\title{
Extração em Fase Sólida Dispersiva na determinação de resíduos e contaminantes em alimentos
}

\author{
Liziara da Costa Cabrera', Manoel Leonardo Martins², Ednei Gilberto Primel', \\ Osmar Damian Prestes², Martha Bohrer Adaime², Renato Zanella ${ }^{2 *}$ \\ ${ }^{1}$ Escola de Química e Alimentos, Universidade Federal do Rio Grande - FURG, Cep 96201-900, Rio Grande, RS, Brasil \\ ${ }^{2}$ Departamento de Química, Universidade Federal de Santa Maria - UFSM, Cep 97105-900, Santa Maria, RS, Brasil \\ e-mail: rzanella@base.ufsm.br
}

\section{Resumo}

Este artigo proporciona uma visão geral da técnica de Extração em Fase Sólida Dispersiva (D-SPE) como uma importante etapa do método QuEChERS que, com um procedimento fácil e rápido, permite obter extratos mais limpos para as análises multirresíduo. A D-SPE envolve, em geral, o uso de um ou mais sorventes, juntamente com sulfato de magnésio anidro, em um tubo de centrífuga, para remover os coextrativos do extrato orgânico. Esta técnica é prática e eficiente para limpar extratos de diferentes amostras complexas, como alimentos de origem vegetal ou animal. Alguns detalhes práticos do uso de diferentes sorventes são apresentados com as principais aplicações.

Palavras-chave

Preparo de amostra; limpeza de extratos; sorventes.

\section{Dispersive Solid Phase Extraction in the determination of residues and contaminants in food}

\section{Abstract}

This paper provide an overview of the technique Dispersive Solid Phase Extraction (D-SPE) as an important step of the QuEChERS method that permit to obtain, in a easy and quick procedure, cleaned extracts for the multiresidue analysis. The D-SPE involves, in general, the use of one or more sorbent together with magnesium sulfate anhydrous in a centrifuge tube to remove the co-extractives from the organic extract. This technique is practical and efficient to clean-up extracts from different complex samples, like foods of vegetable or animal origin. Some practical details of different sorbents are presented with principal applications.

Keywords

Sample preparation; clean-up; sorbents. 


\section{Introdução}

$\mathrm{Na}$ análise de resíduos e contaminantes em alimentos, o preparo de amostra envolve a extração dos analitos e a subsequente limpeza do extrato com a finalidade de isolar os compostos de interesse e remover interferentes da matriz que afetam o sistema de análise. Estes procedimentos consistem em transferir os analitos da matriz para outra fase, empregando simultaneamente uma etapa de limpeza para remoção de substâncias interferentes (isolamento), aumentando a concentração dos analitos (enriquecimento) a um nível acima do limite de detecção da técnica analítica ${ }^{[1]}$. Nos últimos anos, ocorreu um rápido desenvolvimento de novos métodos analíticos, visando à determinação de resíduos e contaminantes em alimentos. Estes avanços tiveram como objetivo a substituição dos métodos tradicionais de análise de resíduos, que apresentavam como características a morosidade de suas diversas etapas, emprego de grandes volumes de solvente, alto custo, etc. ${ }^{[2]}$.

Geralmente, a complexidade da composição dos alimentos ocasiona dificuldades para a quantificação de resíduos e contaminantes, sendo necessária a realização de uma etapa de limpeza do extrato, após extração com solvente. Essa etapa é fundamental, uma vez que reduz as interferências e o efeito matriz, além de diminuir a necessidade de manutenção do sistema cromatográfico. No sentido de melhorar a análise quantitativa, esforços foram realizados no desenvolvimento de novos métodos de extração e limpeza ${ }^{[2]}$. O efeito matriz é observado quando uma considerável diferença de resposta é obtida entre padrões preparados no solvente e aqueles preparados no extrato da matriz, com boa precisão. Uma das maneiras de minimizar e/ou eliminar este efeito é reduzir a quantidade de com- ponentes da matriz que coeluem com os analitos no detector e, para isto, métodos de extração mais seletivos e etapas mais eficientes de limpeza (clean-up) devem ser desenvolvidos. Contudo alternativas, como por exemplo, a padronização externa realizada em extrato da matriz idêntica ou similar ao da amostra é geralmente utilizada ${ }^{[3]}$. $\mathrm{Na}$ Cromatografia Gasosa (Gas Chromatography, GC-MS) o efeito de matriz, além de gerar porcentagens de recuperação superiores a 100\%, pode causar outras alterações na análise cromatográfica, como: i) mascaramento do pico do analito de interesse, gerando um resultado falso negativo; ii) erro na identificação do analito, pois componentes da matriz podem ser erroneamente identificados como o analito de interesse quando, na verdade, este está ausente, gerando resultado falso positivo; iii) aumento no sinal do detector, levando à superestimação do resultado. Isso ocorre quando impurezas voláteis são eluídas com o mesmo tempo de retenção dos analitos de interesse; extinção do sinal no detector $^{[4]}$. Nas análises por Cromatografia Líquida acoplada à Espectrometria de Massas (Liquid Chromatography coupled to Mass Spectrometry, LC-MS), a presença da matriz pode resultar em mudanças na eficiência de ionização, uma vez que pode ocorrer supressão ou aumento da ionização. O mecanismo exato da supressão iônica é desconhecido, porém é causado pela presença de substâncias não voláteis e por compostos com superfície bastante ativa. O efeito matriz também é dependente da natureza química do analito, pois a eficiência de ionização dos compostos polares é mais influenciada pela presença de coextrativos provenientes da matriz quando comparada com compostos apolares. Alguns parâmetros instrumentais, como fonte de ionização e vazão da fase móvel, também influenciam na extensão do efeito matriz ${ }^{[5]}$. 
As limitações na análise de resíduos e contaminantes em alimentos foram minimizadas após o desenvolvimento dos métodos multirresíduo. Estes apresentam como vantagens a possibilidade de analisar um grande número de compostos, altos percentuais de recuperação dos analitos, remoção dos possíveis interferentes da amostra, boa precisão e robustez, baixo custo, rapidez, facilidade e segurança (utilizam pequenos volumes de solventes de baixa toxicidade $)^{[2]}$.

A etapa de limpeza é essencial para promover robustez e confiabilidade aos resultados obtidos pelo sistema cromatográfico, uma vez que componentes não voláteis da matriz podem ficar aderidos no sistema de injeção e também na coluna cromatográfica, alterando a resposta do sistema e aumentando a frequência de manutenções técnicas no equipamento ${ }^{[6,7]}$.

Tradicionalmente a etapa de limpeza nos métodos multirresíduo emprega Extração em Fase Sólida (Solid Phase Extraction, SPE), a qual utiliza cartuchos ou colunas que contêm entre 250 e $1000 \mathrm{mg}$ de sorvente ${ }^{[8]}$. Esta técnica envolve operação manual, uso de diferentes solventes para lavagem do sorvente, etapas de evaporação e secagem. Muitos fatores afetam a precisão quando se trabalha com SPE, entre eles o ajuste do sistema de vácuo e a vazão dos solventes. Esta técnica, quando automatizada, requer manutenção frequente, além dos sistemas hoje disponíveis apresentarem um custo considerável ${ }^{[8]}$. Nos últimos anos, técnicas alternativas foram desenvolvidas com o objetivo de melhorar a etapa de limpeza ${ }^{[9]}$. Este artigo tem como objetivo apresentar algumas aplicações que demonstram a praticidade e a eficiência da Extração em Fase Sólida Dispersiva (Dispersive Solid Phase Extraction, D-SPE) na limpeza de extratos para a determinação de resíduos e contaminantes em alimentos $^{[10-12]}$.

\section{Preparo de amostra para a determinação de resíduos e contaminantes em alimentos}

Nos últimos anos, as técnicas mais amplamente utilizadas para extração de resíduos e contaminantes em alimentos foram a Extração Sólido-Líquido (Solid Liquid Extraction, SLE) e a Extração Líquido-Líquido (Liquid Liquid Extraction, LLE). A SLE e a LLE consistem na extração sucessiva de amostras sólidas e líquidas, respectivamente, com solvente orgânico empregando agitação vigorosa. Estas técnicas têm sido aplicadas para diferentes tipos de amostras, como, por exemplo, alimentos de origem vegetal ou animal ${ }^{[13-15]}$. Em geral, a maioria dessas técnicas emprega solventes orgânicos em alguma de suas etapas. A acetonitrila é considerada um dos melhores solventes, uma vez que promove uma extração bastante eficiente (altos percentuais de recuperação) e extrai baixas concentrações de coextrativos da matriz, sendo eficaz na desnaturação de proteínas e na inativação de enzimas ${ }^{[2]}$. Metanol e acetato de etila também são amplamente utilizados, mas extraem elevadas quantidades de coextrativos da matriz ${ }^{[13-15]}$.

Os avanços da Química Analítica em consonância com o conceito de sustentabilidade levaram ao desenvolvimento de vários métodos de preparo de amostra de alimentos ${ }^{[16]}$. No final da década de 1980, Barker et al. ${ }^{[17]}$ propuseram a Dispersão da Matriz em Fase Sólida (Matrix Solid Phase Dispersion, MSPD), a qual envolve a mistura da amostra (viscosa, sólida ou semissólida) com um sorvente, a transferência da mistura para um cartucho e uma etapa de eluição com um solvente adequado ${ }^{[18,19]}$. Na Extração Ultrassônica (Ultrasonic Extraction, USE), a amostra com solvente é submetida ao banho com ultrassom ${ }^{[20]}$. A USE também tem sido associada a outras técnicas como a MSPD ${ }^{[21]}$. Na Extração Líquida Pressurizada (Pressurized Liquid Extraction, 
PLE), também chamada de Extração Acelerada por Solvente (Accelerated Solvent Extraction, ASE), amostra e solvente são aquecidos e submetidos a alta pressão em uma cela fechada. Com a pressão e temperatura elevadas, muda a viscosidade do solvente, aumentando a extração dos analitos $^{[22]}$. É uma técnica de extração rápida e que reduz consideravelmente a quantidade de solvente ${ }^{[23]}$. Com a otimização de alguns parâmetros como temperatura, pressão e polaridade do solvente é possível obterem boas recuperações com a PLE ${ }^{[24]}$, no entanto as diferentes propriedades físico-químicas dos analitos dificultam o emprego em análises multirresíduo. A Extração Assistida por Micro-ondas (Microwave-Assisted Extraction, MAE) foi utilizada pela primeira vez para extração de compostos orgânicos por Ganzler et al. ${ }^{[25]}$, no entanto se tornou mais popular recentemente. Nesta técnica, a amostra é imersa num frasco aberto ou fechado contendo solvente e é irradiada com micro-ondas. A principal limitação da técnica é o uso de solventes que absorvam energia de micro-ondas, pois solventes não polares não absorvem essa energia ${ }^{[26,27]}$. Além disso, em geral, é necessária uma etapa de limpeza do extrato ${ }^{[28]}$.

Os métodos de preparo de amostra que têm como base a instrumentação, sendo a extração muitas vezes automatizada, demandam analistas treinados e etapas de limpeza entre extrações, o que implica em um maior tempo de análise. Outra desvantagem geralmente apresentada é o escopo limitado de analitos que pode ser extraído em determinadas condições. Sendo assim, estes procedimentos, podem ser empregados em algumas aplicações, mas estão distantes de serem considerados ideais para um método multirresíduo $^{[29]}$.

Anastassiades et al. ${ }^{[10]}$, com o objetivo de superar as limitações práticas dos métodos multirresíduo existentes, introduziram um novo procedimento de preparo de amostra para extração de resíduos e contaminantes denominado QuEChERS (Quick, Easy, Cheap, Effective, Rugged, Safe). Este método tem como vantagem ser rápido, fácil, econômico, efetivo, robusto e seguro, explorando as possibilidades oferecidas pela instrumentação analítica moderna ${ }^{[16]}$. O método QuEChERS pode ser caracterizado como uma sequência das seguintes etapas: Extração (agitação manual ou com auxílio de vórtex da amostra de interesse com acetonitrila, geralmente na proporção 1:1); Partição (adição de sais para promover o efeito salting out, o tamponamento do $\mathrm{pH}$ do meio, além da remoção de água); e Limpeza do extrato (realizada por D-SPE). A versão original deste método apresentou excelentes resultados para diferentes tipos de amostras ${ }^{[16,30,31]}$. Porém, algumas aplicações mostraram que certos compostos apresentavam problemas de estabilidade e/ou recuperação de acordo com o $\mathrm{pH}$ da matriz ${ }^{[11,32]}$. Desta forma, durante o período de otimização do método, percebeu-se que a utilização de tampões de $\mathrm{pH}$ (faixa de 4,8 a 5,0) promovia recuperações satisfatórias (>70\%) para compostos dependentes do $\mathrm{pH}$, independente da matriz utilizada ${ }^{[32]}$.

Atualmente, como o método e suas modificações são aplicados para uma grande variedade de matrizes e para vários compostos, como, por exemplo, agrotóxicos, medicamentos veterinários e micotoxinas, vários sorventes e combinações vêm sendo utilizadas, dependo do tipo de matriz, técnica cromatográfica e dos tipos de compostos analisados. A Figura 1 apresenta as principais etapas para a execução da limpeza de extratos empregando a D-SPE.

\subsection{Extração em fase sólida dispersiva}

A D-SPE, proposta por Anastassiades et al. ${ }^{[10]}$, está baseada em um procedimento muito simples para ser empregado na limpeza de extratos 


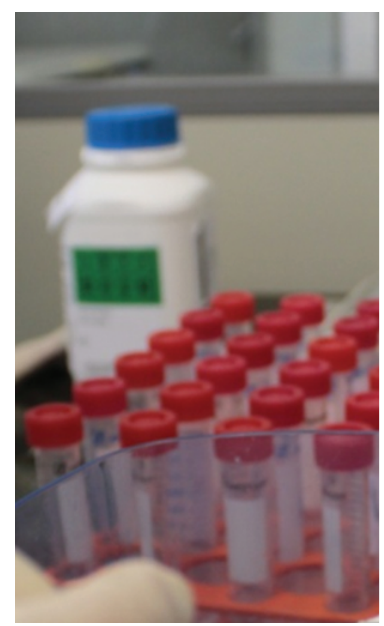

Pesagem do sorvente

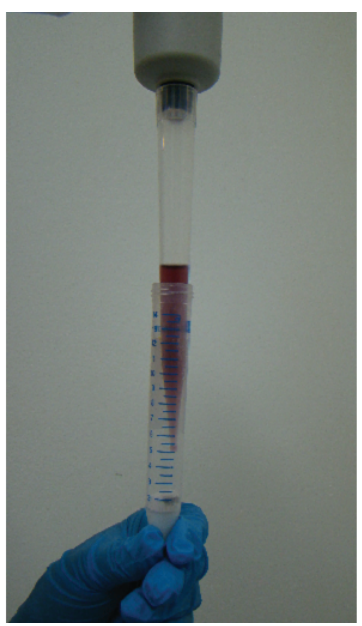

Transferência do extrato

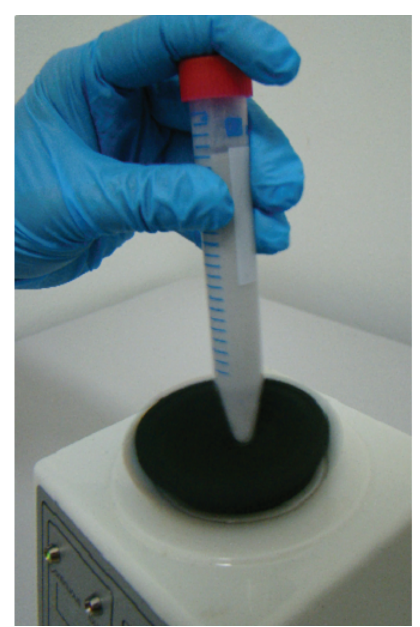

Agitação em vortex

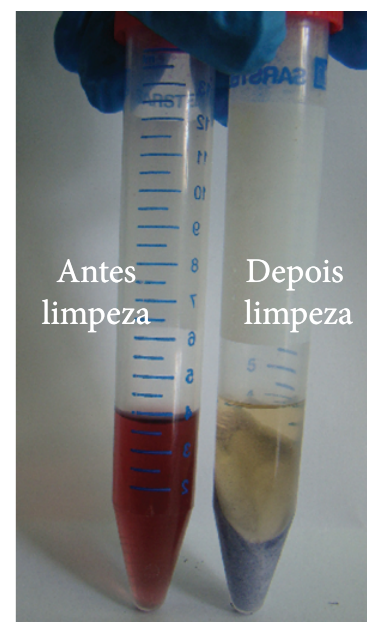

Centrifugação

Figura 1 Representação das etapas de execução da D-SPE.

destinados à análise cromatográfica de resíduos e contaminantes em alimentos. Na proposta original, agita-se o extrato $(1 \mathrm{~mL})$ com pequena quantidade de sorvente ( $25 \mathrm{mg}$ ). A agitação tem como objetivo a distribuição uniforme do sorvente e assim facilitar o processo de limpeza. O sorvente então é separado por centrifugação, sendo uma alíquota do extrato final retirada para análise. Desta maneira, o sorvente atua como um filtro químico, retendo os coextrativos da matriz. Esta técnica pode ser comparada com a MSPD, porém a diferença entre elas esta na adição do sorvente a uma alíquota do extrato e não na amostra original. O custo elevado dos sorventes limita o tamanho da amostra que pode ser usado em MSPD. Assim, a MSPD, quando utilizada, deve garantir a representatividade e homogeneidade da amostra. Por outro lado, a D-SPE está intimamente ligada à etapa de extração e fornece uma alíquota homogênea a partir de uma amostra original de qualquer tamanho. Assim, apenas uma pequena quantidade de sorvente é utilizada.

Quando comparada com a SPE tradicional, a D-SPE apresenta algumas vantagens como: o uso de uma menor quantidade de sorvente e solventes, refletindo em menor custo, além de não haver a necessidade de trabalhar no formato de cartucho. Assim, descartam-se as etapas prévias de pré-condicionamento, sendo necessário apenas um pequeno treinamento dos analistas. Ao contrário do formato em coluna, na D-SPE, todo o sorvente interage igualmente com a matriz. Além disso, outra vantagem desta técnica é a possibilidade de realizar a fácil combinação de diferentes tipos de sorventes de acordo com a necessidade do analista, do tipo de matriz e do equipamento que será utilizado na determinação dos analitos de interesse. Soma-se a isto a possibilidade de realizar a remoção de água residual de forma simultânea com a limpeza do extrato, uma vez que os sais secantes $\left(\mathrm{MgSO}_{4}\right.$ ou $\left.\mathrm{Na}_{2} \mathrm{SO}_{4}\right)$ podem ser adicionados juntamente com o sorvente.

A D-SPE foi desenvolvida simultaneamente com o método QuEChERS, tendo como objetivo a obtenção de um extrato final com menor quantidade de interferentes, aliada a um menor custo quando comparada com técnicas tradicionais. Uma das principais vantagens da D-SPE é a versatilidade no estabelecimento de novos métodos, uma vez que permite a utilização de dife- 
rentes quantidades e/ou misturas de sorventes, dependendo do tipo de matriz e de analitos de interesse.

A seguir, as propriedades e as principais aplicações dos sorventes mais utilizados nesta técnica são detalhadas. A Tabela 1 apresenta aplicações da D-SPE na limpeza de extratos provenientes de diferentes tipos de matrizes de alimentos.

\subsubsection{Amina primária secundária (PSA)}

O sorvente etilenodiamino-N-propil é uma amina primária e secundária (PSA) que atua como um trocador aniônico e pode interagir com outros compostos através de pontes de hidrogênio ou dipolo-dipolo. Possui forte interação com os compostos da matriz, sendo usado para remoção de vários coextrativos interferentes ${ }^{[10]}$. A estrutura bidentada do PSA (Figura 2) tem um elevado efeito quelante, devido à presença dos grupos amino primário e secundário na sua estrutura ${ }^{[12]}$. Como resultado, a retenção de ácidos graxos livres, açúcares e de outros compostos polares presentes na matriz é muito forte, podendo ocorrer a retenção de alguns analitos que interfem no resultado da análise. Por outro lado, não é tão eficiente na remoção de gorduras. O tamanho de partícula do sorvente utilizado é, geralmente, de $40 \mu \mathrm{m}$. O sorvente PSA tem sido usado em associação com outros sorventes, principalmente com $\mathrm{C}_{18}$ ou carbono grafitizado. As diferentes versões do método QuEChERS utilizam, em geral, 25 ou $50 \mathrm{mg}$ de PSA para cada $\mathrm{mL}$ de extrato ${ }^{[12]}$. Para algumas matrizes, como, por exemplo, cereais, utiliza-se uma maior quanti-

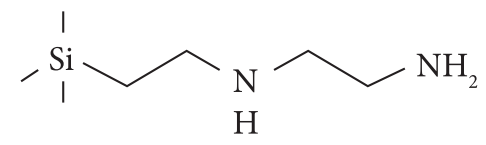

Figura 2 Estrutura do sorvente PSA. dade de PSA na etapa de D-SPE com o intuito de remover, de forma mais eficiente, os ácidos graxos coextraídos ${ }^{[33]}$.

\subsubsection{Octadecilsilano $\left(\mathrm{C}_{18}\right)$}

Uma modificação bastante relevante na etapa de D-SPE foi a adição do sorvente octadecilsilano $\left(\mathrm{C}_{18}\right)$ para promover uma limpeza mais efetiva de algumas matrizes, em especial aquelas que contêm gordura ${ }^{[1,15,33-35]}$. O sorvente $\mathrm{C}_{18}$ é mais utilizado para matrizes com teor de gordura maior que $2 \%$, pois remove com boa eficiência interferentes apolares, tais como substâncias graxas e lipídios ${ }^{[36]}$. No entanto, o sorvente $\mathrm{C}_{18}$ tem sido utilizado em conjunto com PSA para diferentes tipos de matrizes e analitos. O $\mathrm{C}_{18}$ é bastante usado na determinação de compostos orgânicos em matrizes aquosas por SPE e tem um custo inferior ao do PSA. O tamanho de partícula do sorvente $\mathrm{C}_{18}$ mais utilizado é o de $40 \mu \mathrm{m}$, que também é o mais usado em SPE.

\subsubsection{Carbono grafitizado}

A redução do teor de pigmentos nos extratos provenientes de amostras vegetais foi outro avanço efetuado na etapa de limpeza, obtido através da adição de uma pequena quantidade de carvão ativado ou carbono grafitizado ${ }^{[15,34,37]}$. O carvão disponível comercialmente para fins cromatográficos, comumente chamado de carbono grafitizado (graphitized carbon black, GCB), possui uma grande área superficial e contém grupos altamente polares na superfície, com alto potencial para formação de pontes de hidrogênio. Em decorrência destas características, ocorre forte retenção de analitos planares que contenham um ou mais grupos ativos em sua estrutura, resultando em baixas recuperações para estes com$\operatorname{postos}^{[35,38]}$. Entre os compostos planares que 
Tabela 1 Sorventes utilizados em D-SPE e detalhes das aplicações.

\begin{tabular}{|c|c|c|c|}
\hline Matriz & Analitos & D-SPE: $\mathrm{mg}$ de sorvente $/ \mathrm{mL}$ de extrato & Ref. \\
\hline Cevada & herbicidas & 25 mg PSA & 48 \\
\hline Cebola, limão, tomate e uva & agrotóxicos & 25 mg PSA & 49 \\
\hline Repolho e rabanete & agrotóxicos & 25 mg PSA & 50 \\
\hline Frutas & $\mathrm{OCP}$ & 25 mg PSA & 51 \\
\hline Suco de cana-de-açúcar & agrotóxicos & $50 \mathrm{mg}$ PSA & 52 \\
\hline Frango & $\begin{array}{l}\text { medicamentos } \\
\text { veterinários }\end{array}$ & 150 mg PSA & 53 \\
\hline Arroz & fenoxiácidos & $250 \mathrm{mg}$ PSA & 39 \\
\hline Trigo, farinha e farelo & agrotóxicos & $125 \mathrm{mg} \mathrm{C}_{18}$ & 54 \\
\hline Ervilha e lima & agrotóxicos & $12 \mathrm{mg}$ PSA + 3,75 mg GCB & 15 \\
\hline Molho de maçã-mirtilo & agrotóxicos & $25 \mathrm{mg}$ PSA $+25 \mathrm{mg} \mathrm{C}_{18}$ & 15 \\
\hline Vegetais e & agrotóxicos & $50 \mathrm{mg}$ PSA + 100, 120 ou $140 \mathrm{mg} \mathrm{C}_{18}$ & \\
\hline alimentos infantis & & $\begin{array}{l}\text { para matrizes com } 2,3 \text { e } 4,5 \% \text { de gordura, } \\
\text { respectivamente }\end{array}$ & 55 \\
\hline \multirow[t]{4}{*}{ Mel, própolis e geleia real } & quinolonas & $37,5 \mathrm{mg} \mathrm{C}_{18}$ & \\
\hline & & $37,5 \mathrm{mg} \mathrm{C}_{18}+37,5 \mathrm{mg}$ PSA & 56 \\
\hline & & $100 \mathrm{mg} \mathrm{C}_{18}+100 \mathrm{mg}$ PSA & \\
\hline & & $100 \mathrm{mg} \mathrm{C}_{18}+100 \mathrm{mg}$ PSA + $100 \mathrm{mg}$ GCB & \\
\hline $\begin{array}{l}\text { Repolho, espinafre, uva e } \\
\text { laranja }\end{array}$ & agrotóxicos & 10 mg nanotubos de carbono & 47 \\
\hline $\begin{array}{l}\text { Berinjela, pepino, couve e } \\
\text { pimenta }\end{array}$ & OPP & 16 mg nanotubos de carbono & 27 \\
\hline \multirow[t]{2}{*}{ Azeitonas e azeite de oliva } & OCP, OPP & PSA, GCB e $C_{18}$ & 57 \\
\hline & triazinas & & \\
\hline Plantas medicinais & $\mathrm{OCP}$ & $16,7 \mathrm{mg}$ PSA + 33,4 mg GCB & 58 \\
\hline Sangue & drogas & $41,7 \mathrm{mg} \mathrm{C}_{18}+41,7 \mathrm{mg}$ PSA & 59 \\
\hline
\end{tabular}

apresentam baixa recuperação quando GCB é empregado na etapa de D-SPE, pode-se destacar hexaclorobenzeno ${ }^{[39]}$, terbufós e tiabendazol ${ }^{[40]}$.

\subsubsection{Outros sorventes}

Koesukwiwat et al. ${ }^{[39]}$ testaram a extração de agrotóxicos fenoxiácidos, como $2,4 \mathrm{D}$, quincloraque, picloram e outros, por QuEChERS modificado, utilizando como sorvente na D-SPE alumina combinada com $\mathrm{C}_{18}$. A alumina ou óxido de alumínio $\left(\mathrm{Al}_{2} \mathrm{O}_{3}\right)$ tem características alcalinas, embora possa também ser preparada para apresentar características neutra ou ácida. É geralmente empregada na separação cromatográfica de compostos lipofílicos e, pelo fato de poder ser preparada com características ácida, neutra ou alcalina, é bastante útil na separação de substâncias que apresentem variações dessas características.

Nos métodos mais antigos, o florisi ${ }^{\circledR}$ era bastante empregado, sendo utilizado na forma de colunas recheadas, nas quais os extratos das 
amostras eram percolados. Neste tipo de procedimento, empregava-se um maior volume de solventes orgânicos, sucessivas percolações no leito de sorvente e etapas posteriores de concentração dos extratos. Nguyen et al. ${ }^{[41]}$ utilizaram florisil ${ }^{\circledR}$ na etapa de D-SPE para limpeza de amostra de óleo de soja, visando a determinação de resíduos de 95 agrotóxicos. Em cromatografia, o florisil ${ }^{\circledR}$ é utilizado para separação de analitos com baixa polaridade.

A terra diatomácea, também denominada diatomita, diatomita calcinada, diatomita fluxo calcinada, sílica diatomácea e sílica amorfa, destaca-se pelo seu baixo custo, alta área superficial e baixa massa específica, tendo como componente majoritário a sílica, a qual se encontra na forma hidratada. A coloração varia do branco ao cinza-escuro e o tamanho de partícula varia de 4 e $500 \mu \mathrm{m}$. A terra diatomácea também foi utilizada em métodos de preparo de amostra empregando MSPD para extração de agrotóxicos ${ }^{[19]}$. Cabrera $^{[42]}$ testou terra diatomácea (branca, de 64-76 $\mu \mathrm{m}$ de tamanho de partícula) como sorvente na D-SPE, empregando QuEChERS modificado, para 26 agrotóxicos em arroz, entretanto, somente 9 compostos apresentaram recuperações dentro da faixa de $70 \%$ a $120 \%$ e, na mistura com $\mathrm{C}_{18}, 14$ compostos ficaram dentro dessa faixa de recuperação.

Quitosana é um polissacarídeo catiônico produzido através da desacetilação da quitina, um polissacarídeo encontrado no exoesqueleto de crustáceos, através de um processo de alcalinização sob altas temperaturas. Quitina e quitosana são biopolímeros renováveis, de baixo custo e suscetíveis a alterações químicas para aumentar sua capacidade de adsorção ${ }^{[43]}$. Cabrera ${ }^{[42]}$ obteve bons resultados de recuperação para 20 de 26 agrotóxicos testados em arroz, empregando D-SPE com quitosana e QuEChERS modificado.
Nos últimos anos, sorventes alternativos estão sendo utilizados na D-SPE com a finalidade de obterem melhores resultados na etapa de limpeza dos extratos gerados pelo método QuEChERS. Como alternativa para minimizar as perdas de analitos provocadas pelo sorvente GCB, tem-se o sorvente polimérico ChloroFiltr ${ }^{\circledast}$, que remove de maneira seletiva a clorofila, não afetando as recuperações de compostos plana$\operatorname{res}^{[44]}$.

Zhao et al. ${ }^{[45]}$ sintetizaram um novo sorvente para D-SPE a partir de tetraetilenopentamina. $\mathrm{O}$ potencial de limpeza do novo material é comparável com o do PSA. Este sorvente remove com eficiência pigmentos, ácidos orgânicos e açúcares. Além disso, outra vantagem deste novo material é a possibilidade de reutilização por mais de 5 vezes sem perder a eficiência de limpeza. Os autores obtiveram recuperações entre $75 \%$ e $114 \%$ (RSD $\leq 17 \%$ ) para 29 agrotóxicos (organoclorados e organofosforados). Os resultados mostraram o potencial de aplicação deste novo material, uma vez que a precisão e a seletividade do método proposto foram satisfatórias para análise de resíduos de agrotóxicos em alimentos.

Devido à sua área superficial extremamente grande, os nanotubos de carbono possuem grande capacidade de adsorção. Assim, estão sendo utilizados como sorventes alternativos em D-SPE. Do ponto de vista estrutural, classificam-se em: nanotubos de carbono de parede simples, que podem ser considerados como uma única folha de grafite enrolada sobre si mesma para formar um tubo cilíndrico; e os nanotubos de carbono de parede múltipla, que compreendem um conjunto de nanotubos concêntricos ${ }^{[46]}$. A utilização de nanotubos de carbono de paredes múltiplas está tendo sucesso na etapa de D-SPE para determinação multirresíduo de agrotóxicos em frutas e vegetais. Zhao et al. ${ }^{[4]}$ utilizaram o 
método QuEChERS empregando nanotubos de carbono de paredes múltiplas como sorvente na etapa de D-SPE. Este método foi aplicado para determinação multirresíduo de agrotóxicos em vegetais por GC-MS. Os resultados de recuperação foram de $71 \%$ a $110 \%$ (RSD $\leq 15 \%)$. O método apresentou linearidade na faixa entre 20 e $500 \mu \mathrm{g} \mathrm{L}^{-1}$. Os limites de quantificação e os limites de detecção variaram de 3 a $50 \mu \mathrm{g} \mathrm{kg}^{-1} \mathrm{e}$ 1 a $20 \mu \mathrm{g} \mathrm{kg}^{-1}$, respectivamente. O método foi aplicado com sucesso em amostras reais sendo considerado confiável e robusto. Os nanotubos de carbono de paredes múltiplas apresentam-se como uma alternativa ao sorvente PSA, uma vez que os métodos que empregam estes sorventes apresentam resultados satisfatórios nas análises de resíduos e contaminantes em alimentos. Recentemente, Zhao et al. ${ }^{[27]}$ utilizaram nanotubos de carbono na etapa de D-SPE para determinação de agrotóxicos em vegetais. A D-SPE com nanotubos de carbono foi comparada com $\mathrm{C}_{18}$, PSA e GCB. A limpeza dos extratos obtida com o uso de nanotubos de carbono foi mais eficiente e foram obtidas melhores recuperações dos analitos e menor efeito de matriz. Esse material já tinha sido utilizado na limpeza de extratos empregando cartuchos SPE, também com excelentes resultados.

\subsection{Combinação entre D-SPE e outras técnicas}

Nos últimos anos, o método QuEChERS iniciou uma verdadeira transformação no preparo de amostra para análise de resíduos de agrotóxicos em alimentos, uma vez que suas características de simplicidade, rapidez, baixo custo, entre outras, foram incorporadas em vários laboratórios de rotina. Porém, ao ser comparado com métodos comumente utilizados para análise de resíduos de agrotóxicos em alimentos, observa-se que a relação entre a quantidade de amos- tra e de solvente $\left(1 \mathrm{~g} \mathrm{~mL}^{-1}\right)$ obtida no método QuEChERS é baixa se comparada com os valores típicos de 2 a $5 \mathrm{~g} \mathrm{~mL}^{-1}$ dos métodos que utilizam solventes apolares ${ }^{[60]}$. A fim de minimizar esta limitação, Melo et al. ${ }^{[61]}$ realizaram a combinação entre os extratos provenientes da etapa de D-SPE com a técnica de Microextração LíquidoLíquido Dispersiva (Dispersive Liquid-Liquid Microextraction, DLLME) na análise de resíduos de agrotóxicos por HPLC-DAD. Depois da etapa de extração com acetonitrila, seguida da partição promovida pelos sais e limpeza por D-SPE, os analitos foram concentrados em triclorometano pelo procedimento DLLME. Esta combinação resultou em procedimento simples, rápido e barato, além de proporcionar baixos limites de detecção $\left(1,7\right.$ a $\left.45 \mu \mathrm{g} \mathrm{kg}{ }^{-1}\right)$ para os 13 compostos avaliados. A combinação D-SPE/DLLME e HPLC-DAD também foi empregada para determinação de 7 inseticidas neonicotinóides em amostras de arroz, painço e aveia ${ }^{[62]}$. As vantagens da utilização em conjunto da D-SPE com DLLME incluem a versatilidade, baixo custo, tempo de análise relativamente curto e baixo consumo de solventes orgânicos. Além disso, destacam-se elevada eficiência de extração e efeito matriz pouco significativo. Neste método, na etapa de D-SPE, empregaram-se os sorventes PSA, GCB e $\mathrm{C}_{18}$. Os resultados apresentaram boa linearidade $\left(\mathrm{r}^{2} \geq 0,99\right)$, recuperações satisfatórias (76\% a $123 \%)$, e RSD\% aceitáveis ( $\leq 12,6 \%)$. Os limites de detecção obtidos estavam entre 2 e $5 \mu \mathrm{gg}^{-1}$ e os limites de quantificação entre $7 \mathrm{e}$ $18 \mu \mathrm{g} \mathrm{kg}$.

Satpathy et al. ${ }^{[26]}$ desenvolveram um método multirresíduo para análise de 72 agrotóxicos em frutas e vegetais empregando extração por MAE, limpeza do extrato por D-SPE seguida de determinação por GC-MS. Os resultados apresentaram boa linearidade, robustez, precisão e reprodutibilidade. Esta combinação permite que 
a etapa de extração seja automatizada, tornando este método uma ferramenta atrativa para a análise de rotina de agrotóxicos em frutas e vegetais. A combinação MAE e D-SPE também foi avaliada na determinação de 27 agrotóxicos por GC-MS em amostras de ervas medicinais ${ }^{[63]}$. A técnica D-SPE simplificou o processo de limpeza e minimizou o consumo de solventes orgânicos. Os limites de detecção e quantificação variaram entre 0,2 e $10 \mu \mathrm{g} \mathrm{kg}^{-1}$ e entre 0,8 e $30 \mu \mathrm{g} \mathrm{kg}^{-1}$, respectivamente. As recuperações de todos os analitos variaram entre $70 \%$ e $120 \%$ com os $\mathrm{RSD} \leq 17,2 \%$, atendendo aos requisitos para análise de rotina de resíduos de agrotóxicos.

A limpeza do extrato à baixa temperatura (freeze-out ou low temperature clean-up), visando a redução dos coextrativos lipídicos, tem sido empregada com sucesso na determinação de diversos compostos em diferentes matrizes. A técnica consiste na precipitação de extrativos lipídicos, dentre outros, que são congelados junto com a água, sendo retirado o extrato composto pelo solvente extrator. A combinação desta técnica com D-SPE, reduz ainda mais a quantidade de coextrativos ${ }^{[15,35]}$, possibilitando um menor consumo de sorventes. No entanto, uma das principais desvantagens do congelamento é o tempo, o qual varia, geralmente, de 30 minutos a 12 horas ou mais. A maioria dos estudos utiliza mais de 12 horas ${ }^{[64-67]}$. Contudo, quando combinado com D-SPE ou outra técnica de limpeza, o tempo necessário para a limpeza do extrato pode ser reduzido como demonstrado por Koesukwiwat et al. ${ }^{[35]}$ que, em 2 horas de resfriamento, conseguiram uma adequada redução de coextrativos. Depois do congelamento foi realizada D-SPE com PSA e $\mathrm{C}_{18}$. Chen et al. ${ }^{[68]}$, na análise de pesticidas organoclorados em amostras de peixe, congelaram o extrato por 20 minutos, realizando em seguida a limpeza em cartucho SPE recheado com aminopropil. Os limites de detecção do método variaram de 0,5 a $20 \mu \mathrm{g} \mathrm{kg}^{-1}$, e as recuperações entre 81,3 e 113,7\% com RSD $\leq 13,5 \%$. Hong et al. ${ }^{[69]}$, também analisando organoclorados em peixe, utilizaram a técnica de limpeza à baixa temperatura, congelando o extrato por 30 minutos, seguida de SPE com florisil $^{\oplus}$ e obtiveram bons resultados. Os percentuais de recuperação foram $\geq 80 \%$. Os limites de detecção variaram de 0,5 a $5 \mu \mathrm{g} \mathrm{kg}^{-1}$, exceto para endossulfam que foi de $20 \mu \mathrm{g} \mathrm{kg}$.

\section{Conclusões e perspectivas futuras}

Nos últimos anos, várias técnicas de preparo de amostra foram desenvolvidas com o objetivo de reduzir etapas e/ou minimizar o consumo de solventes. Entre estas novas possibilidades, o método QuEChERS está ganhando popularidade na análise de resíduos e contaminantes em alimentos. Esta grande aceitação está relacionada com os resultados satisfatórios obtidos no preparo de amostra de diferentes matrizes contendo analitos com propriedades físico-químicas distintas. A D-SPE tem mostrado ser uma excelente técnica de limpeza de extratos, sua versatilidade, rapidez e baixo custo, quando comparada a outras técnicas, fazem dela uma excelente alternativa. Salienta-se que os resultados obtidos por esta técnica estão intimamente relacionados com o uso da espectrometria de massas. Trabalhos publicados recentemente mostram que a D-SPE pode ser utilizada em combinação com outras técnicas de extração. Assim, esta técnica de limpeza apresenta um futuro promissor na análise de resíduos e contaminantes, podendo estar associada ou não com o método que lhe deu origem.

\section{Referências}

1 Kinsella B, O’Mahony J, Malone E, Moloney M, Cantwell H, Furey A et al. Current trends in sample 
preparation for growth promoter and veterinary drug residue analysis. Journal of Chromatography A 2009; 1216(46):7977-8015. http://dx.doi. org/10.1016/j.chroma.2009.09.005

2 Prestes OD, Friggi CA, Adaime MB, Zanella R. QuEChERS - Um método moderno de preparo de amostra para determinação multirresíduo de pesticidas em alimentos por métodos cromatográficos acoplados à espectrometria de massas. Química Nova 2009; 32:1620-1634. http://dx.doi.org/10.1590/ S0100-40422009000600046

3 Picó Y, Blasco C, Font G. Environmental and food applications of LC-tandem mass spectrometry in pesticide-residue analysis: An overview. Mass Spectrometry Reviews 2004; 23:45-85. PMid:14625892.

4 Pinho GP, Neves AA, Queiroz MELR, Silverio FO. Efeito de matriz na quantificação de agrotóxicos por cromatografia gasosa. Química Nova 2009; 32:987-95. http://dx.doi.org/10.1590/S0100-40422009000400030

5 Kruve A, Künnapas A, Herodes K, Leito I. Matrix effects in pesticide multi-residue analysis by liquid chromatography-mass spectrometry. Journal of Chromatography A 2008; 1187(1-2):58-66. http:// dx.doi.org/10.1016/j.chroma.2008.01.077

6 Saito Y, Kodama S, Matsunaga A, Yamamoto A. Multiresidue Determination of Pesticides in Agricultural Products by Gas Chromatography/ Mass Spectrometry with Large Volume Injection. Journal of AOAC International 2004; 87:1356-67. PMid:15675447.

7 Ueno E, Oshima H, Saito I, Matsumoto H, Yoshimura Y, Nakazawa H. Multiresidue Analysis of Pesticides in Vegetables and Fruits by Gas Chromatography/Mass Spectrometry after Gel Permeation Chromatography and Graphitized Carbon Column Cleanup. Journal of AOAC International 2004; 87(4):1003-15. PMid:15295897.

8 Hyötyläinen T. On-line coupling of extraction with gas chromatography. Journal of Chromatography A 2008; 1186(1-2):39-50. http://dx.doi.org/10.1016/j. chroma.2007.11.066

9 Lambropoulou DA, Albanis TA. Methods of sample preparation for determination of pesticide residues in food matrices by chromatography-mass spectrometry-based techniques: a review. Analytical and Bioanalytical Chemistry 2007; 389(6):1663-83. http://dx.doi.org/10.1007/s00216-007-1348-2

10 Anastassiades M, Lehotay SJ, Stajnbaher D, Schenck FJ. Fast and easy multiresidue method employing acetonitrile extraction/partitioning and "dispersive solid-phase extraction" for the determination of pesticide residues in produce. Journal of AOAC International 2003; 86(2):412-31. PMid:12723926.

11 Lehotay SJ, Mastovská K, Lightfield AR. Use of Buffering and Other Means to Improve Results of Problematic Pesticides in a Fast and Easy Method for Residue Analysis of Fruits and Vegetables. Journal of AOAC International 2005; 88:615. PMid:15859090.

12 Prestes OD, Adaime MB, Zanella R. QuEChERS: possibilidades e tendências no preparo de amostra para determinaçãomultirresíduodepesticidasemalimentos. Scientia Chromatographica 2011; 3(1):51-64. http:// dx.doi.org/10.4322/sc.2011.004

13 Marazuela MD, Bogialli S. A review of novel strategies of sample preparation for the determination of antibacterial residues in foodstuffs using liquid chromatography-based analytical methods. Analytica Chimica Acta 2009; 645(1-2):5-17. PMid:19481624.

14 LeDoux M. Analytical methods applied to the determination of pesticide residues in foods of animal origin. A review of the past two decades. Journal of Chromatography A 2011; 1218:1021-36. http://dx.doi. org/10.1016/j.chroma.2010.12.097

15 Lehotay SJ, Son KA, Kwon H, Koesukwiwat U, Fu W, Mastovska K et al. Comparison of QuEChERS sample preparation methods for the analysis of pesticide residues in fruits and vegetables. Journal of Chromatography A 2010; 1217(16):2548-60. PMid:20144460. http://dx.doi.org/10.1016/j. chroma.2010.01.044

16 Ridgway K, Lalljie SPD, Smith RM. Sample preparation techniques for the determination of trace residues and contaminants in foods. Journal of Chromatography A 2007; 1153(1-2):36-53. http://dx.doi.org/10.1016/j. chroma.2007.01.134

17 Barker SA, Long AR, Short CR. Isolation of drug residues from tissues by solid phase dispersion. Journal of Chromatography A 1989; 475(2):353-61. http://dx.doi.org/10.1016/S0021-9673(01)89689-8

18 Barker SA. Applications of matrix solid-phase dispersion in food analysis. Journal of Chromatography A 2000; 880(1-2):63-8. http://dx.doi.org/10.1016/ S0021-9673(99)01290-X

19 Rodrigues SA, Caldas SS, Primel EG. A simple, efficient and environmentally friendly method for the extraction of pesticides from onion by matrix solidphase dispersion with liquid chromatography-tandem mass spectrometric detection. Analytica Chimica Acta 2010; 678(1):82-9. http://dx.doi.org/10.1016/j. aca.2010.08.026 
20 Rezić I, Horvat AJM, Babić S, Kaštelan-Macan M. Determination of pesticides in honey by ultrasonic solvent extraction and thin-layer chromatography. Ultrasonics Sonochemistry 2005; 12(6):477-81. http:// dx.doi.org/10.1016/j.ultsonch.2004.07.004

21 García-Valcárcel AI, Tadeo JL. A combination of ultrasonic assisted extraction with LC-MS/MS for the determination of organophosphorus pesticides in sludge. Analytica Chimica Acta 2009; 641:117-23. PMid:19393375.

22 Carabias-Martínez R, Rodríguez-Gonzalo E, Revilla-Ruiz P, Hernández-Méndez J. Pressurized liquid extraction in the analysis of food and biological samples. Journal of Chromatography A 2005; 1085(1-2):1-17. http://dx.doi.org/10.1016/j. chroma.2005.06.072

23 Chuang JC, Hart K, Chang JS, Boman LE, Van Emon JM, Reed AW. Evaluation of analytical methods for determining pesticides in baby foods and adult duplicate-diet samples. Analytica Chimica Acta 2001; 444:87. http://dx.doi.org/10.1016/S00032670(01)01164-3

24 Cho SK, El-Aty AMA, Jeon HR, Choi JH, Shin HC, Shim JH. Comparison of different extraction methods for the simultaneous determination of pesticide residues in kiwi fruit using gas chromatography-mass spectrometry. Biomedical Chromatography 2008; 22:727-35. http://dx.doi. org/10.1002/bmc.990

25 Ganzler K, Salgó A, Valkó K. Microwave extraction: A novel sample preparation method for chromatography. Journal of Chromatography A 1986; 371:299-306. http://dx.doi.org/10.1016/S0021-9673(01)94714-4

26 Satpathy G, Tyagi YK, Gupta RK. A novel optimised and validated method for analysis of multiresidues of pesticides in fruits and vegetables by microwave-assisted extraction (MAE)-dispersive solid-phase extraction (d-SPE)-retention time locked (RTL)-gas chromatography-mass spectrometry with Deconvolution reporting software (DRS). Food Chemistry 2011; 127:1300-08. http://dx.doi. org/10.1016/j.foodchem.2011.01.087

27 Zhao X, Xu X, Su R, Zhang H, Wang Z. An application of new microwave absorption tube in non-polar solvent microwave-assisted extraction of organophosphorus pesticides from fresh vegetable samples. Journal of Chromatography A 2012; 1229:6-12. http://dx.doi. org/10.1016/j.chroma.2012.01.018

28 Chen L, Ding L, Jin H, Song D, Zhang H, Li J et al. The determination of organochlorine pesticides based on dynamic microwave-assisted extraction coupled with on-line solid-phase extraction of high-performance liquid chromatography. Analytica Chimica Acta 2007; 589:239-46. http://dx.doi.org/10.1016/j. aca.2007.03.003

29 Wardencki WM, Curylo J. A review of theoretical and practical aspects of solid-phase microextraction in food analysis. International Journal of Food Science and Technology 2004; 39:703-17. http://dx.doi. org/10.1111/j.1365-2621.2004.00839.x

30 Lehotay SJ, Hiemstra M, Bodegraven P, Kok A. Validation of a fast and easy method for the determination of residues from 229 pesticides in fruits and vegetables using gas and liquid chromatography and mass spectrometric detection. Journal of AOAC International 2005; 88:595-614. PMid:15859089.

31 Quick Easy Cheap Effective Rugged Safe. [cited 2012 Jun]. Available from: http:www.quechers.com.

32 Payá $\mathrm{P}$, Anastassiades M, Mack D, Sigalova I, Tasdelen B, Oliva J et al. Analysis of pesticide residues using the Quick Easy Cheap Effective Rugged and Safe (QuEChERS) pesticide multiresidue method in combination with gas and liquid chromatography and tandem mass spectrometric detection. Analytical and Bioanalytical Chemistry 2007; 389:1697-714. http:// dx.doi.org/10.1007/s00216-007-1610-7

33 Mastovska K, Dorweiler KJ, Lehotay SJ, Wegscheid JS, Szpylka KA. Pesticide Multiresidue Analysis in Cereal Grains Using Modified QuEChERS Method Combined with Automated Direct Sample Introduction GC-TOFMS and UPLC-MS/MS Techniques. Journal of Agricultural and Food Chemistry 2010; 58:5959-72. PMid:20028018.

34 Cunha SC, Lehotay SJ, Mastovska K, Fernandes JO, Oliveira BMPP. Evaluation of the QuEChERS sample preparation approach for the analysis of pesticide residues in olives. Journal of Separation Science 2007; 30:620-32. PMid:17444232.

35 Koesukwiwat U, Lehotay SJ, Mastovska K, Dorweiler KJ, Leepipatpiboon N. Extension of the QuEChERS Method for Pesticide Residues in Cereals to Flaxseeds, Peanuts, and Doughs. Journal of Agricultural and Food Chemistry 2010; 58:5950-8. PMid:20025276.

36 Lehotay S. Determination of Pesticide Residues in Foods by Acetonitrile Extraction and Partitioning with Magnesium Sulfate: Collaborative Study. Journal of the Association of Official Analytical Chemists 2007; 90:485-520. PMid:17474521. 
37 Anastassiades M, Scherbaum E, Tasdelen B, Stajnbaher D. Crop protection, public health, environmental safety. Weinheim, Germany: Wiley-VCH; 2007.

38 Hennion MC. Graphitized carbons for solidphase extraction. Journal of Chromatography A 2000; 885(1-2):73-95. http://dx.doi.org/10.1016/ S0021-9673(00)00085-6

39 Koesukwiwat U, Sanguankaew K, Leepipatpiboon $\mathrm{N}$. Rapid determination of phenoxy acid residues in rice by modified QuEChERS extraction and liquid chromatography-tandem mass spectrometry. Analytica Chimica Acta 2008; 626:10-20. PMid:18761116.

40 Wilkowska A, Biziuk M. Determination of pesticide residues in food matrices using the QuEChERS methodology. Food Chemistry 2011; 125:803-12. http://dx.doi.org/10.1016/j.foodchem.2010.09.094

41 Nguyen TD, Lee MH, Lee GH. Rapid determination of 95 pesticides in soybean oil using liquid-liquid extraction followed by centrifugation, freezing and dispersive solid phase extraction as cleanup steps and gas chromatography with mass spectrometric detection. Microchemical Journal 2010; 95:113-9. http://dx.doi.org/10.1016/j.microc.2009.11.009

42 Cabrera L. Estudo do efeito matriz na determinação de agrotóxicos em diferentes tipos de arroz pelo método QuEChERS modificado e LC-MS/MS. [tese]. Santa Maria: Universidade Federal de Santa Maria; 2012.

43 Dotto GL, Vieira MLG, Gonçalves JO, Pinto LAdA. Remoção dos corantes azul brilhante, amarelo crepúsculo e amarelo tartrazina de soluções aquosas utilizando carvão ativado, terra ativada, terra diatomácea, quitina e quitosana: estudos de equilíbrio e termodinâmica. Química Nova 2011; 34:1193-9. http://dx.doi.org/10.1590/S0100-40422011000700017

44 QuEChERS ChloroFiltr. [cited 2012 Jun]. Available from: http://unitedchem.com/product.aspx?P=276.

45 Zhao YG, Shen HY, Shi JW, Chen XH, Jin MC. Preparation and characterization of amino functionalized nano-composite material and its application for multi-residue analysis of pesticides in cabbage by gas chromatography-triple quadrupole mass spectrometry. Journal of Chromatography A 2011; 1218(33):5568-80. http://dx.doi.org/10.1016/j. chroma.2011.06.090

46 Herbst MH, Macedo MIF, Rocco AM. Tecnologia dos nanotubos de carbono: tendências e perspectivas de uma área multidisciplinar. Química Nova 2004; 27:986-92. http://dx.doi.org/10.1590/ S0100-40422004000600025
47 Zhao P, Wang L, Zhou L, Zhang F, Kang S, Pan C. Multi-walled carbon nanotubes as alternative reverseddispersive solid phase extraction materials in pesticide multi-residue analysis with QuEChERS method. Journal of Chromatography A 2012; 1225:17-25. http:// dx.doi.org/10.1016/j.chroma.2011.12.070

48 Díez C, Traag WA, Zommer P, Marinero P, Atienza J. Comparison of an acetonitrile extraction/partitioning and "dispersive solid-phase extraction" method with classical multi-residue methods for the extraction of herbicide residues in barley samples. Journal of Chromatography A 2006; 1131(1-2):11-23. http:// dx.doi.org/10.1016/j.chroma.2006.07.046

49 Lesueur C, Knittl P, Gartner M, Mentler A, Fuerhacker M. Analysis of 140 pesticides from conventional farming foodstuff samples after extraction with the modified QuECheRS method. Food Control 2008; 9:906-14. http://dx.doi.org/10.1016/j. foodcont.2007.09.002

50 Nguyen TD, Yu JE, Lee DM, Lee G-H. A multiresidue method for the determination of 107 pesticides in cabbage and radish using QuEChERS sample preparation method and gas chromatography mass spectrometry. Food Chemistry 2008; 110:207-213. http://dx.doi.org/10.1016/j.foodchem.2008.01.036

51 Cieślik E, Sadowska-Rociek A, Ruiz JMM, Surma-Zadora M. Evaluation of QuEChERS method for the determination of organochlorine pesticide residues in selected groups of fruits. Food Chemistry 2011; 125:773-8. http://dx.doi. org/10.1016/j.foodchem.2010.09.019

52 Furlani RPZ, Marcilio KM, Leme FM, Tfouni SAV. Analysis of pesticide residues in sugarcane juice using QuEChERS sample preparation and gas chromatography with electron capture detection. Food Chemistry 2011; 126:1283-7. http://dx.doi. org/10.1016/j.foodchem.2010.11.074

53 Lopes RP, Reyes RC, Romero-González R, Frenich AG, Vidal JLM. Development and validation of a multiclass method for the determination of veterinary drug residues in chicken by ultra high performance liquid chromatography-tandem mass spectrometry. Talanta 2012; 89:201-8. http://dx.doi.org/10.1016/j. talanta.2011.11.082

54 Kolberg DI, Prestes OD, Adaime MB, Zanella R. Development of a fast multiresidue method for the determination of pesticides in dry samples (wheat grains, flour and bran) using QuEChERS based method and GC-MS. Food Chemistry 2011; 125:1436-42. http://dx.doi.org/10.1016/j.foodchem.2010.10.041 
55 Georgakopoulos P, Zachari R, Mataragas M, Athanasopoulos P, Drosinos EH, Skandamis PN. Optimisation of octadecyl $\left(\mathrm{C}_{18}\right)$ sorbent amount in QuEChERS analytical method for the accurate organophosphorus pesticide residues determination in low-fatty baby foods with response surface methodology. Food Chemistry 2011; 128:536-42. http://dx.doi.org/10.1016/j.foodchem.2011.03.042

56 Lombardo-Agüí M, García-Campaña AM, GámizGracia L, Cruces-Blanco C. Determination of quinolones of veterinary use in bee products by ultrahigh performance liquid chromatography-tandem mass spectrometry using a QuEChERS extraction procedure. Talanta 2012; 93:193-9. http://dx.doi. org/10.1016/j.talanta.2012.02.011

57 García-Reyes JF, Ferrer C, Gómez-Ramos MJ, Fernández-Alba AR, Molina-Díaz A. Determination of pesticide residues in olive oil and olives. Trends in Analytical Chemistry 2007; 26:239-51. http://dx.doi. org/10.1016/j.trac.2007.01.004

$58 \mathrm{Xu} \mathrm{R}, \mathrm{Wu}$ J, Liu Y, Zhao R, Chen B, Yang M et al. Analysis of pesticide residues using the Quick Easy Cheap Effective Rugged and Safe (QuEChERS) pesticide multiresidue method in traditional Chinese medicine by gas chromatography with electron capture detection. Chemosphere 2011; 84:908-12. http://dx.doi.org/10.1016/j.chemosphere.2011.06.013

59 Usui K, Hayashizaki Y, Hashiyada M, Funayama M. Rapid drug extraction from human whole blood using a modified QuEChERS extraction method. Legal Medicine 2012. http://dx.doi.org/10.1016/j. legalmed.2012.04.008

60 Hiemstra M, De Kok A. Comprehensive multiresidue method for the target analysis of pesticides in crops using liquid chromatography-tandem mass spectrometry. Journal of Chromatography A 2007; 1154:3-25. http://dx.doi.org/10.1016/j. chroma.2007.03.123

61 Melo A, Mansilha C, Pinho O, Ferreira IMPLVO. Analysis of Pesticides in Tomato Combining QuEChERS and Dispersive Liquid-Liquid Microextraction Followed by High-Performance Liquid Chromatography. Food Analytical Methods 2012. In press. http://dx.doi.org/10.1007/ s12161-012-9469-4

62 Wang P, Yang X, Wang J, Cui J, Dong AJ, Zhao HT et al. Multi-residue method for determination of seven neonicotinoid insecticides in grains using dispersive solid-phase extraction and dispersive liquid-liquid micro-extraction by high performance liquid chromatography. Food Chemistry 2012, 134:1691-8. http://dx.doi.org/10.1016/j.foodchem.2012.03.103

63 Mao X, Wan Y, Yan A, Shen M, Wei Y. Simultaneous determination of organophosphorus, organochlorine, pyrethriod and carbamate pesticides in Radix astragali by microwave-assisted extraction/dispersivesolid phase extraction coupled with GC-MS. Talanta 2012, 97:131-41. http://dx.doi.org/10.1016/j. talanta.2012.04.007

64 Lentza-Rizos C, Avramides EJ, Kokkinaki K. Residues of azoxystrobin from grapes to raisins. Journal of Agricultural and Food Chemistry 2006; 54:138-41. PMid:16390190.

65 Rübensam G, Barreto F, Hoff RB, Pizzolato TM. Determination of avermectin and milbemycin residues in bovine muscle by liquid chromatography-tandem mass spectrometry and fluorescence detection using solvent extraction and low temperature cleanup. Food Control 2013; 29:55-60. http://dx.doi.org/10.1016/j. foodcont.2012.05.075

66 Goulart SM, Alves RD, Neves AA, De Queiroz JH, De Assis TC, De Queiroz M. Optimization and validation of liquid-liquid extraction with low temperature partitioning for determination of carbamates in water. Analytica Chimica Acta 2010; 671:41-7. http://dx.doi. org/10.1016/j.aca.2010.05.003

67 Goulart S, Dequeiroz M, Neves A, Dequeiroz J. Low-temperature clean-up method for the determination of pyrethroids in milk using gas chromatography with electron capture detection. Talanta 2008; 75:1320-23. http://dx.doi.org/10.1016/j. talanta.2008.01.058

68 Chen S, Yu X, He X, Xie D, Fan Y, Peng J. Simplified pesticide multiresidues analysis in fish by low-temperature cleanup and solid-phase extraction coupled with gas chromatography/mass spectrometry. Food Chemistry 2009; 113:1297-300. http://dx.doi. org/10.1016/j.foodchem.2008.08.045

69 Hong J, Kim HY, Kim DG, Seo J, Kim KJ. Rapid determination of chlorinated pesticides in fish by freezing-lipid filtration, solid-phase extraction and gas chromatography-mass spectrometry. Journal of Chromatography A 2004; 1038:27-35. http://dx.doi. org/10.1016/j.chroma.2004.03.003 